\title{
Stress, coping mechanisms and quality of life in hemodialysis patients
}

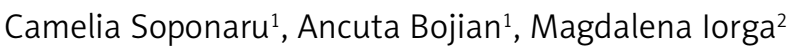

${ }^{1}$ University Alexandru Ioan Cuza of Iasi, Iasi, Romania

${ }^{2}$ Dialysis Unit, St Mary University Hospital of Iasi, University of Medicine

and Pharmacy „Gr.T.Popa”, lasi, Romania

Submitted: 21 March 2016

Accepted: 7 April 2016

Arch Med Sci Civil Dis 2016; 1: e16-e23

DOI: 10.5114/amscd.2016.59601

Copyright @ 2016 Termedia \& Banach

\section{Abstract}

Introduction: The aims of the study were to identify the impact of coping mechanisms on quality of life in hemodialysis (HD) patients and to establish the associations between stress factors and coping mechanisms, to evaluate coping mechanisms as significant predictors of physical and mental health, and to identify the effects of demographic and socioeconomic factors on coping mechanisms.

Material and methods: The study included a group of $70 \mathrm{HD}$ patients, $38.6 \%$ males and $61.4 \%$ females, mean age of $54.55 \pm 11.70$. Data were collected by means of the Hemodialysis Stressors Scale for estimating stress, the Jalowiec Coping Scale (JCS) for identifying coping mechanisms, and the Short Form 36 Health Survey Questionnaire for assessing quality of life (QoL).

Results: The frequency of using coping mechanisms is: optimism $(M=2.26$ $\pm 0.48)$, support $(M=2.16 \pm 0.57)$, confrontational coping $(M=1.96 \pm 0.47)$, one's own strength $(M=1.94 \pm 0.46)$, palliative coping $(M=1.44 \pm 0.54)$, emotive coping $(M=1.40 \pm 0.61)$, evasive coping $(M=1.39 \pm 0.46)$, fatalism $(M=1.23 \pm 0.74)$. Emotion-focused coping correlates with the following stressors: role ambiguity $(r=0.33 ; p=0.004)$, dependence on staff $(r=0.25$, $p=0.03)$ and daily activity $(r=0.27, p=0.02)$.

Conclusions: Optimism is the most commonly used defense mechanism, assessed as the most effective one. The fatalistic coping mechanism is a significant predictor of mental health, while optimism and palliative coping are significant predictors of physical health.

Key words: quality of life, stress, patient, dialysis, coping mechanism.

\section{Introduction}

Stress has been traditionally regarded as a response, a stimulus, and, more recently, a transaction. Stress is usually seen as being a state of emergency, in contrast to everyday situations of adaptation. It refers both to a person's daily experiences and to challenges or difficult life events. Coping represents a person's cognitive and behavioral efforts to manage (reduce, minimize, control or tolerate) internal or external claims of the transaction between the person and the environment which are perceived as demanding or exceeding personal resources. Coping has two major functions: facing the problem that causes distress (problem-focused coping) and emotion adjustment (emotion-focused coping). Other authors postulate that people resort to a combination of the two

\author{
Corresponding author: \\ Magdalena lorga \\ Dialysis Unit \\ St Mary University \\ Hospital of lasi \\ University of Medicine \\ and Pharmacy „Gr.T.Popa” \\ 7 Universitatii St \\ 700460 lasi, Romania \\ Phone: +40722800936 \\ E-mail: magdaiorga@ \\ yahoo.com
}


types of coping mechanisms in order to face most threatening situations, and they support the idea that there are no superior or inferior coping mechanisms, the degree of adequacy or effectiveness being determined by the effects of these mechanisms in a particular context [1].

The dominance of a particular type of coping strategy is determined by contextual factors, such as duration, severity and type of stressor, but also by personal factors such as temperament. The chronic kidney disease patient will face the physiological symptoms of the disease, but also the associated changes at the level of social life and cognitive functioning [2]. In addition to the above, hemodialysis (HD) patients, compared to individuals from the general population, experience intense states of anxiety, depression and low life expectations [3-6].

In the early stages of the disease, the patient may only need rest and dietary restrictions, but, as the disease progresses, he may suffer major losses and significant changes at the level of lifestyle [7]. A non-exhaustive taxonomy places hemodialytic stressors in two categories: that of physiological stressors, and that of psychosocial stressors. In the category of stressors of a physical nature, we encounter dependence on the dialysis machine, functional limitations, altered sexual function, food and drink constraints, the ingestion of numerous drugs in order to treat the disease, and loss of appetite and energy [8, 9]. Psychosocial stressors are represented by time constraints, fear of death, loss of employment and autonomy, and changes at the level of self-perception [10]. We may also include the restriction of social contacts in the category of psychosocial factors.

Postulating a unanimously accepted definition of the concept of quality of life is difficult, because it includes multiple dimensions. Quality of life represents the individual's subjective perception of his life in the context of the culture and values of the system in which he lives, in relation to his objectives, expectations, standards and preoccupations [11]. The relation between hemodialytic stressors and hemodialysis patients' quality of life is mediated by coping processes. The effects of dialysis on these patients' quality of life are important, and some studies have demonstrated the existence of a negative correlation between emotion-focused coping and the total score for quality of life. However, no association between problem-focused coping and subjects' quality of life was identified. This result suggests that, regardless of how often patients resort to strategies of problem-focused coping, their quality of life is not affected. As long as the problem remains, it is difficult for an improvement in the quality of life to take place. Thus, patients diagnosed with end- stage kidney disease must learn to live with the implications of this diagnosis and find effective coping strategies [12-15].

Hemodialysis is a treatment method that is a source of stress, even if the main purpose is to prolong life [16]. In this study we demonstrate that the mechanisms of coping (as an independent variable) used by HD patients to deal with sources of stress are related to hemodialysis (as an independent variable) as a treatment which ensures their existence and their influence on quality of life (as a dependent variable).

\section{Aim}

I) Identifying the effect of coping mechanisms on the level of quality of life in hemodialysis patients (the extent to which physical and mental health are influenced by coping mechanisms). II) Identifying significant predictors of physical and mental health among the eight coping strategies. III) Ranking the coping mechanisms depending on their frequency of use, but also on their effectiveness. IV) Identifying the impact of the interaction between stress and coping mechanisms on quality of life (physical and mental health) in hemodialysis patients. V) Highlighting the relationship between stressors: daily activity, physical symptoms, dependence on medical staff, food and drink restrictions, role ambiguity, vascular access, functioning of the reproductive system and the two types of coping mechanisms, problem-focused and emotion-focused. VI) Identifying significant correlations between the seven stressors specific to hemodialysis and coping mechanisms: confrontational, evasive, optimistic, fatalistic, emotive, palliative, support and one's own strength. VII) Observing the differences in what concerns coping mechanisms adopted by hemodialysis patients depending on the studied demographic variables.

\section{Material and methods}

The research involved the evaluation of $70 \mathrm{pa}-$ tients from an urban dialysis center for adults from North-Eastern Romania, with ages from 19 to 76 years old $(M=54.55 \pm 11.70)$. Of the 70 patients diagnosed with stage 5 chronic kidney disease, $38.6 \%$ were male and $61.4 \%$ were female; $52.9 \%$ lived in urban environments, while $42.9 \%$ lived in rural environments. By level of education, $14.3 \%$ had no more than 8 years of schooling, $54.9 \%$ had schooling from the $9^{\text {th }}$ to the $12^{\text {th }}$ grade, and $30 \%$ had over 12 years of schooling. Of the surveyed subjects, $14.3 \%$ were single and $85.7 \%$ were part of a couple. The selection of participants was based on a number of criteria, such as: they had started the hemodialytic treatment of renal function replacement at least 6 months before, were at 
least 18 years old, and had the cognitive ability to understand the meaning of items of the research instruments and give an answer according to their subjective feelings.

The sampling method used was for convenience, non-random, being justified by subjects' specific features, but also by the researcher's resources. The process of data collection took place from March 2014 to December 2014. Patients were given an informed consent form, by which they were made aware of the purpose of the research, the instructions for filling in the instruments used throughout the study, the principles of data privacy, as well as the possibility to withdraw at any moment from the group of subjects, without repercussions of any kind, especially those connected to the quality of health services they benefit from.

There were two ways to fill in the instruments: as an interview conducted by the researcher, or by self-administration. In the first situation, filling in the surveys took place during hemodialysis sessions, while the self-administration of instruments entailed their completion by subjects at home, after being given instructions for it.

The instrument used during this research was made of four parts. The first section aimed at identifying demographic data such as age, gender, marital status, level of education and original environment.

The second section is represented by three scales: The Hemodialysis Stressor Scale, the Short Form 36 (SF-36) Survey and the Jalowiec Coping Scale translated, adapted and validated for the research using the Cronbach's $\alpha$ coefficient for scales and subscales.

The Hemodialysis Stressor Scale as an instrument. By means of this scale, we assessed the incidence and intensity of stressors associated with the hemodialytic treatment of stage 5 chronic kidney disease. This scale comprises 31 items forming the following dimensions: daily activity, physical symptoms, dependence on medical staff, food and drink restrictions, role ambiguity, vascular access and functioning of the reproductive system. The subjects assessed, on a four-stage scale, the intensity with which each stressor acted upon them $(0=$ not at all stressful, $1=$ a little stressful, 2 = moderately stressful, 3 = very stressful). The total score is calculated by adding up scores obtained for the 31 items; there is a linear relationship between stress intensity and the value of the total score. The internal consistency of this scale was proved by means of Cronbach's $\alpha$ coefficient, both for the entire instrument $(\alpha=0.915)$ and for each of the seven dimensions.

The Short Form 36 (SF-36) Survey is a generic instrument for measuring quality of life. This is an instrument validated on the general, "healthy" Romanian population, as well as on iteratively hemodialyzed patients. The instrument comprises 36 items, 8 scales aggregating items (physical function, physical role, somatic pain, overall health, vitality, social function, emotional role and mental health) and two generic concepts aggregating the scales (physical health and mental health). In this study, the value of the Cronbach's $\alpha$ coefficient for the 36 items was 0.82, while for the "physical health" dimension, $\alpha=0.72$, and for the "mental health" dimension the Cronbach's $\alpha$ coefficient had a value of 0.72 . The most important limitation of this instrument is that it does not cover two important dimensions: sexual activity and sleep.

The Jalowiec Coping Scale was applied in order to identify the coping mechanisms used by hemodialysis patients to face hemodialytic stressors. This comprises 60 items and two parts, A and B. Part $A$ of the instrument measures the frequency with which subjects use coping mechanisms, while part $B$ helps assess the usefulness of the mechanisms adopted by subjects in their fight against a specific stressor. Part A of the Jalowiec instrument obtained an Cronbach's $\alpha$ coefficient of 0.85 , while for part B, the Cronbach's $\alpha$ coefficient was 0.94 . The $\alpha$ coefficient was also calculated for the eight dimensions of the instrument, presented in Table I, the data being presented in the table below. In order to obtain a more accurate image of the internal consistency of the eight subscales of the instrument, we also identified the value of the mean correlation between items.

\section{Statistical analysis}

To attain the objectives of this research, we used the SPSS software for statistical analysis. The normal distribution of variables was assessed by means of the Kolmogorov-Smirnov test. In order to test the extent to which the type of coping mechanisms influences hemodialysis patients' quality of life (physical and mental health) the $t$ test for independent samples was applied. In order to evaluate the degree of prediction of the variables confrontational coping, evasive coping, optimistic coping, emotive coping, palliative coping, support coping, coping by one's own strength for quality of life we performed sixteen simple linear regressions. To test the interaction effect between the stress variable and the coping mechanisms on hemodialysis patients' quality of life, the factorial ANOVA technique was applied. The identification of significant associations between the coping mechanisms and stress factors was performed by calculating the Pearson correlation coefficient. In order to detect differences caused by demographic variables, taken into account within the study, concerning coping mechanisms, 
Table I. Internal consistency of the Jalowiec Coping Scale

\begin{tabular}{|c|c|c|c|c|}
\hline \multirow[t]{2}{*}{ Scale } & \multicolumn{2}{|c|}{ Part A } & \multicolumn{2}{|c|}{ Part B } \\
\hline & Cronbach's $\alpha$ & $\begin{array}{c}\text { Mean of inter-item } \\
\text { correlation }\end{array}$ & Cronbach's $\alpha$ & $\begin{array}{c}\text { Mean of inter-item } \\
\text { correlation }\end{array}$ \\
\hline Confrontational & 0.69 & 0.21 & 0.82 & 0.35 \\
\hline Evasive & 0.67 & 0.15 & 0.76 & 0.22 \\
\hline Optimistic & 0.61 & 0.25 & 0.81 & 0.45 \\
\hline Fatalistic & 0.43 & 0.20 & 0.25 & 0.14 \\
\hline Emotive & 0.62 & 0.25 & 0.52 & 0.17 \\
\hline Palliative & 0.60 & 0.20 & 0.75 & 0.35 \\
\hline Support & 0.58 & 0.22 & 0.64 & 0.27 \\
\hline Based on one's own strength & 0.54 & 0.15 & 0.73 & 0.25 \\
\hline Full scale & 0.85 & & 0.94 & \\
\hline
\end{tabular}

three $t$ tests for independent samples and a oneway ANOVA were carried out.

\section{Results}

\section{Objective I}

The first objective of this study was to test the effect of coping mechanisms on the variables physical and mental health. The independent variable "coping mechanisms" has two levels, that is, problem-focused coping mechanisms and emotion-focused coping mechanisms. In line with the statistical results obtained, no significant differences could be observed depending on the type of coping mechanisms used, concerning physical health $[t(68)=0.631, p=0.530]$ or mental health $[t(68)=0.020, p=0.984]$.

The mean value obtained in the "physical health" variable by subjects using emotion-focused coping is 51.88 , while the mean for subjects who primarily adopt problem-focused coping in the same variable is 50.91. The mean value for subjects using emotion-focused coping in the "mental health" variable is 48.91, and that of subjects using problem-focused coping in the same variable is 48.88 .

\section{Objective II}

In order to evaluate the degree of prediction of the variables confrontational coping, evasive coping, optimistic coping, emotive coping, palliative coping, support coping, and coping by one's own strength for physical and mental health, sixteen simple linear regressions were performed.

For mental health, only one significant predictor has been identified: the fatalistic coping mechanism, which explains up to $17.7 \%\left(r^{2}=0.177\right.$, $p<0.001)$ of the criterion variance.

Conversely, the significant predictors of physical health are the optimistic and palliative cop- ing mechanisms, which explain $10.6 \%\left(r^{2}=0.106\right.$, $p=0.006)$ and $12.1 \%\left(r^{2}=0.121, p=0.003\right)$ of the criteria variance.

\section{Objective III}

To achieve the objective regarding the ranking of coping mechanisms according to the frequency of their use, new variables have been made by calculating the arithmetic mean of items forming each scale of the Jalowiec instrument. For ranking the new variables, we calculated the mean and the standard deviation.

According to the statistical data, the most frequently used coping mechanism is optimism $(M=$ $2.26 \pm 0.48)$, followed, in second place, by support $(M=2.16 \pm 0.57)$, in third place, by confrontational coping $(M=1.96 \pm 0.47)$, while the fourth place belongs to one's own strength $(M=1.94 \pm 0.46)$, in fifth place is palliative coping $(M=1.44 \pm 0.54)$, the sixth place is taken by emotive coping $(M=1.40$ \pm 0.61 ), the evasive coping mechanism takes seventh place $(M=1.39 \pm 0.46)$, and the least adopted coping mechanism is fatalism $(M=1.23 \pm 0.74)$.

The top 10 positions were taken by 11 items of the JCS instrument, the item "You have thought of the good things in your life" being in first place $(M=2.62 \pm 0.64)$, "You have wished for the problem to disappear" ranking second $(M=2.50$ \pm 0.79 ), while the item "You have prayed and put your trust in God" ( $M=0.48 \pm 0.86)$ ranked third in the hierarchy. Thus, a first conclusion may be drawn, that subjects of this study most often use emotion-focused coping mechanisms. Nevertheless, of the 11 items ranking in the top 10 positions in the hierarchy of items selected as representatives of the top 10 coping strategies used, 6 items represent problem-focused coping mechanisms, and 5 items represent emotion-focused coping mechanisms. 
Hence the question "Which of the two types of coping mechanisms (emotion-focused and problem-focused) is predominantly used by the subjects of this research?" The answer to the question above is that subjects in this study predominantly use emotion-focused coping $(M=56.70 \pm 11.67)$ rather than problem-focused mechanism $(M=$ $43.21 \pm 8.01)$. Of the 10 items, the ones with the lowest mean were: "You have dreamed of a better life" (position 8; $M=2.35 \pm 0.86$ ), "You have tried to see the bright side of the situation" (position 9; $M=2.30 \pm 0.66$ ), "You have accepted the situation, because few things could be done about it" $(M=$ $2.28 \pm 0.80$ ) and "You have tried to keep your sense of humor" (position 10; $M=2.28 \pm 0.80$ ) (Table II).

After identifying the most frequently adopted coping mechanisms, we wanted to investigate their effectiveness. Thus, we calculated the arithmetic mean of items making up each scale in part B of the Jalowiec Coping Scale. It is important to mention that the effectiveness of seven of the eight coping mechanisms was assessed, the motivation for this being the lack of internal consistency of the scale for assessing fatalistic coping, from the second part of the Jalowiec instrument. The second step was to calculate the mean and standard deviation of the new variables. Apart from the fact that optimism is the most used mechanism of defense against stressors, it turns out that it is considered, by the subjects of this study, the most effective as well $(M=2.17 \pm 0.69)$. The next places are taken, in descending order, by the following mechanisms: support $(M=2.04 \pm 0.63)$, confrontational $(M=1.74 \pm 0.65)$, one's own strength $(M=1.67 \pm 0.59)$, palliative $(M=1.48 \pm 0.71)$, evasive $(M=1.20 \pm 0.59)$ and, the last by frequency, the emotive coping mechanism $(M=0.98 \pm 0.63)$.

\section{Objective IV}

In order to capture the interaction effect of the variables stress and coping mechanisms on the quality of life of hemodialysis patients, we applied the factorial ANOVA method. The statistical results show no interaction effect for the variables stress and coping mechanisms on the variables physical health $[F(1,69)=0.28, p=0.59]$ and mental health $[F(1,69)=2.50, p=0.11]$.

\section{Objective V}

Fulfillment of the fifth objective requires the study of the relation between the stressors daily activity, physical symptoms, dependence on medical staff, food and drink restrictions, role ambiguity, vascular access, functioning of the reproductive system and the two types of coping mechanisms, problem-focused and emotion-focused. To achieve this objective, we calculated the Pearson correlation coefficient (see Table III).

In the table above, one may notice that there are three statistically significant correlations. One of them is that between the variable role ambiguity and emotion-focused coping mechanisms $(r=0.33, p=0.004)$. This correlation may be interpreted thus: when patients' level of stress determined by role ambiguity rises, patients tend to use coping mechanisms aimed at controlling emotions. Moreover, dependence on medical staff correlates with the emotion-focused coping mechanism $(r=0.25, p=0.03)$. This is a positive, statistically significant and low-intensity correlation. Last, but not least, there is a statistically significant correlation between daily activity and emotion-focused coping mechanisms $(r=0.27$, $p=0.02)$.

Table II. Table of items ranked in the top 10, based on the mean

\begin{tabular}{|lrcc|}
\hline $\begin{array}{l}\text { Item } \\
\text { no. }\end{array}$ & Item & Mean & $\begin{array}{c}\text { Standard } \\
\text { deviation }\end{array}$ \\
\hline 1 & SCJ47: You have thought of the good things in your life & 2.62 & 0.64 \\
\hline 2 & SCJ58: You have wished for the problem to disappear & 2.50 & 0.79 \\
\hline 3 & SCJ17: You have prayed and put your trust in God & 0.48 & 0.86 \\
\hline 4 & SCJ16: You have tried to keep the situation under control & 2.45 & 0.71 \\
\hline 5 & SCJ30: You have tried to live a normal life and have not let the problem get in the way & 2.38 & 0.72 \\
\hline 6 & SCJ13: You have tried to look at the problem objectively and to see all its aspects & 2.37 & 0.78 \\
\hline 7 & SCJ11: You have discussed the problem with family or friends & 2.35 & 0.83 \\
\hline 8 & SCJ14: You have dreamed of a better life & 2.35 & 0.86 \\
\hline 9 & SCJ54: You have tried to see the bright side of the situation & 2.30 & 0.66 \\
\hline 10 & SCJ12: You have accepted the situation, because few things could be done about it & 2.28 & 0.80 \\
\hline & SCJ39: You have tried to keep your sense of humor & 2.28 & 0.80 \\
\hline
\end{tabular}


Table III. Table of Pearson correlations between the two types of coping mechanisms and other variables

\begin{tabular}{|lcc|}
\hline Stressor & Emotion-focused coping & Problem-focused coping \\
\hline Daily activity & $r=0.27, p=0.02$ & $r=0.21, p=0.07$ \\
\hline Physical symptoms & $r=0.21, p=0.08$ & $r=0.23, p=0.053$ \\
\hline Dependence on medical staff & $r=0.25, p=0.03$ & $r=0.10, p=0.40$ \\
\hline Food and drink restrictions & $r=0.21, p=0.07$ & $r=0.16, p=0.17$ \\
\hline Role ambiguity & $r=0.33, p=0.004$ & $r=0.07, p=0.53$ \\
\hline Vascular access & $r=0.16, p=0.17$ & $r=0.12, p=0.31$ \\
\hline Functioning of the reproductive system & $r=0.01, p=0.88$ & $r=-0.01, p=0.90$ \\
\hline
\end{tabular}

\section{Objective VI}

By calculating the Pearson correlation coefficient between the 7 specific stressors (confrontational, evasive, optimistic, fatalistic, emotive, palliative, support, one's own strength) and the 8 defense mechanisms studied within this research (daily activity, physical symptoms, dependence on the medical staff, food and drink restrictions, role ambiguity, vascular access and functioning of the reproductive system), we identified the following statistically significant results:

- the physical symptoms stressor positively and significantly correlates with the confrontational coping mechanism, the association between the two variables being weak $(r=0.24, p=0.04)$;

- the daily activity stressor has a positive, weak and statistically significant association with the confrontational $(r=0.25, p=0.03)$ and the evasive coping mechanism $(r=0.26, p=0.02)$. This relation may transpose both the positive and the negative scores of the two variables to a directly proportional relation. Thus, the more the stress generated by daily activity increases, the more patients tend to adopt confrontational and evasive coping as a defense strategy;

- the stressor food and drink restrictions correlates positively, weakly and statistically significantly with the evasive coping mechanism $(r=0.24$, $p=0.04$ );

- the role ambiguity stressor correlates positively and statistically significantly with the evasive coping mechanism, the association between the two variables being of medium intensity $(r=0.40, p=0.001)$;

- the fatalistic coping mechanism correlates statistically significantly and positively with the following stressors: daily activity $(r=0.29$, $p=0.01)$, dependence on medical staff $(r=0.34$, $p=0.004)$ and role ambiguity $(r=0.36, p=$ 0.002 ). Where $r<0.30$, the association between variables is of low intensity, and if the value of $r$ is within the range $0.30-0.60$, the association between variables is of medium intensity.

\section{Objective VII}

Finally, there are no differences regarding the coping mechanisms adopted by hemodialysis patients according to the studied demographic variables: gender $[t(68)=-0.83, p=0.40]$, marital status [ $t(68)=0.93, p=0.43]$, environment $[t(65)$ $=-0.21, p=0.83]$, or level of education $[F(2,65)$ $=2.31, p=0.10]$.

\section{Discussions}

The significant predictors of quality of life are the optimistic, palliative and fatalistic coping mechanisms. Of these three significant predictors, the first two are positive. However, the predictors may also have negative valences, as is the case with the fatalistic coping mechanism. Palliative coping entails the individual's involvement in a number of activities aimed at a short-term improvement in the distress experienced after facing a situation perceived as threatening. In this case, the hemodialysis patient resorting to this coping mechanism takes medicine for stress reduction, eats and drinks more than usual, uses various relaxation techniques, and performs physical activities. This coping mechanism is a positive predictor of physical health.

Mental health is one of the two dimensions of quality of life. In the earlier stages of this research, palliative coping proved to have an influence on physical health (the second dimension of quality of life), as assessed by means of the SF-36 instrument. Palliative coping explains $17.7 \%$ of mental health for hemodialysis patients in the group of subjects included in this study.

The results emphasized the fact that the optimistic coping mechanism was used to a significant and predominant extent, the confrontational and support styles ranking second and third by frequency. The least frequently used coping mechanisms were the emotive, evasive, the one based on one's own strength, fatalistic and palliative one. Optimism was also identified as the most frequently used coping strategy for patients 
diagnosed with chronic kidney disease, who did not undergo dialysis as a form of treatment. Other coping strategies that reached a high frequency were the confrontational, support and the one based on one's own strength, while the emotive and fatalistic coping strategies were recorded as low-frequency [17]. Also a positive attitude and a sense of humor enable patients to lower their level of stress and anxiety [18-20].

The support offered by personal, professional and spiritual support systems is assessed as also being highly effective in facing challenges which occur along with hemodialysis. Thus, debating the implications of the disease with a professional (a doctor, nurse, priest, counselor), discussing this topic with family or friends, sharing one's subjective experience with a person in the same situation or turning toward faith have proved effective in reducing the tension generated by internal and/or external challenges perceived as threatening.

When the level of stress of the "daily activity" dimension rises, patients tend to use the confrontational, evasive and/or fatalistic coping mechanism equally, in order to reduce the tension experienced. Subjects seek strategies for direct confrontation of the situation, they may feel hopeless or they engage in activities aimed at avoiding the stressor. Undergoing hemodialysis sessions several times a week for a long time, patients develop an involuntary dependence on medical staff. In this context, dependence becomes a stressor. In line with the statistical results, when the level of stress generated by the addiction to medical staff rises, the frequency of use of the fatalistic coping mechanism rises to the same extent.

Our study proves that patients predominantly use emotion-focused coping. Beside the fact that the optimistic coping mechanism is the most frequently used mechanism of defense against stressors, it turns out that it is also considered to be the most effective. Demographic variables have not resulted in differences concerning the coping mechanisms used by hemodialysis subjects.

The study presents some limits. They may be explained by cultural characteristics and the small number of participants questioned from a specific geographical area. The items refer to very personal issues and have an important emotional impact. These intrusive questions could influence subjects' answers. On the other hand, the tendency to present a façade causes distortions of some answers. Generally speaking, patients tend to present to others a positive image of themselves. Recognizing their needs is seen as vulnerability, being hard to accept or to disclose to others.

In conclusion, optimism is the most common coping mechanism, and is also rated by subjects as the most effective. It is a significant predictor of mental health, while the coping mechanisms optimism and palliative coping seem to be significant predictors of physical health. No statistically significant differences in coping mechanisms were identified depending on demographic and socioeconomic factors.

\section{Conflict of interest}

The authors declare no conflict of interest.

\section{References}

1. Folkman S, Lazarus RS, Dunkel-Schetter C, DeLongis A, Gruen RJ. Dynamics of a stressful encounter: cognitive appraisal, coping, and encounter outcomes. J Pers Soc Psychol 1986; 50: 992-1003.

2. Moulton A. Chronic kidney disease: the diagnosis of a "unique" chronic disease. CANNT J 2008; 18: 34-8.

3. Feroze U, Martin D, Kalantar-Zadeh K, Kim JC, ReinaPatton A, Kopple JD. Anxiety and depression in maintenance dialysis patients: preliminary data of a cross-sectional study and brief literature review. J Ren Nutr 2012; 22: 207-10.

4. Farrokhi F, Abedi N, Beyene J, Kurdyak P, Jassal SV. Association between depression and mortality in patients receiving long-term dialysis: a systematic review and meta-analysis. Am J Kidney Dis 2014; 63: 623-35.

5. Preljevic VT, Østhus TBH, Sandvik L, et al. Screening for anxiety and depression in dialysis patients: comparison of the Hospital Anxiety and Depression Scale and the Beck Depression Inventory. J Psychosom Res 2012; 73: 139-44.

6. Theofilou P. Quality of life and mental health in hemodialysis and peritoneal dialysis patients: the role of health beliefs. Int Urol Nephrol 2012; 44: 245-53.

7. Shinde M, Mane SP. Stressors and the coping strategies among patients undergoing hemodialysis. Int J Sci Res 2014; 3: 266-76.

8. Calvin AO. Haemodialysis patients and endof life decisions: a theory of personal preservation. J Adv Nurs 2004; 46: 558-66.

9. lorga M, Starcea M, Munteanu M, Sztankovszky LZ. Psychological and social problems of children with chronic kidney disease. Eur J Sci Theol 2014; 10: 179-88.

10. Symister P, Friend R. Quality of life and adjustment in renal disease: a health psychology perspective. In: Health psychology through the life span: practice and research opportunities. Resnick RJ, Rozensky RH (eds.). American Psychological Association, Washington, DC, US 1996; 265-87.

11. Group TW. The World Health Organization quality of life assessment (WHOQOL): development and general psychometric properties. Soc Sci Med 1998; 46: 1569-85.

12. Kimmel PL. Depression in patients with chronic renal disease: what we know and what we need to know. J Psychosom Res 2002; 53: 951-6.

13. Wyld M, Morton RL, Hayen A, Howard K, Webster AC. A systematic review and meta-analysis of utility-based quality of life in chronic kidney disease treatments. PLoS Med 2012; 9: e1001307.

14. Tjaden LA, Grootenhuis MA, Noordzij M, Groothoff JW. Health-related quality of life in patients with pediatric onset of end-stage renal disease: state of the art and recommendations for clinical practice. Pediatr Nephrol 2015; DOI 10.1007/s00467-015-3186-3. 
15. Birmelé B, Le Gall A, Sautenet B, Aguerre C, Camus V. Clinical, sociodemographic, and psychological correlates of health-related quality of life in chronic hemodialysis patients. Psychosomatics 2012; 53: 30-7.

16. Parvan K, Ahangar R, Hosseini FA, et al. Coping methods to stress among patients on hemodialysis and peritoneal dialysis. Saudi J Kidney Dis Transplant 2015; 26 : 255-62.

17. Harwood L, Wilson B, Sontrop J, Clark AM. Chronic kidney disease stressors influence choice of dialysis modality. J Adv Nurs 2012; 68: 2454-65.

18. Molzahn A, Sheilds L, Bruce A, et al. Case study: a model patient or impression management? Nephrol Nurs 2011; 38: 511-3.

19. Perricone G, Guerra MP, Cruz O, et al. Maternal coping strategies in response to a child's chronic and oncological disease: a cross-cultural study in Italy and Portugal. Pediatr Rep 2013; 5: 43-7.

20. Cann A, Collette C. Sense of humor, stable affect, and psychological well-being. Eur J Psychol 2014; 10: 464-79. 